\title{
TEOREMA KONVOLUSI PADA TRANSFORMASI FOURIER FRAKSIONAL QUATERNION SISI KIRI DAN SIFAT-SIFATNYA
}

\author{
N. H. Wibowo', S. Musdalifah², dan Resnawati ${ }^{3}$ \\ 1,2,3Program studi Matematika Jurusan Matematika FMIPA Universitas Tadulako \\ Jalan Soekarno-Hatta Km. 09 Tondo, Palu 94118, Indonesia. \\ 1nofiantohariwibowo@yahoo.com, 2selvymusdalifah@yahoo.com, 3r35n4w4t1@yahoo.com
}

\begin{abstract}
Fourier transform (FT) is growing very rapidly and applying in various fields such as analyzing and decomposing signals in the frequency domain. FT has been extended to quaternion algebra known as the Quaternion Fourier Transform (QFT). The purpose of this paper are to formulate the definition and properties of the left sided Quaternion Fractional Fourier Transform (QFFT), to formulate the definition and convolution theorem for left sided QFFT. Firstly, the results showed the formulation of the left sided QFFT definition and some of the properties such as linearity, translation, modulation and scalar. Secondly, its showed the formulation of convolution theorem for left sided QFFT and also the left sided QFFT of conjugate and translation convolution.
\end{abstract}

\section{Keywords : : Algebra Quaternion, Convolution Theorem, Fourier Transform, Fractional Fourier Transform Quaternion.}

\section{ABSTRAK}

Transformasi Fourier (TF) berkembang sangat pesat dan diterapkan di berbagai bidang seperti menganalisa dan mendekomposisi sinyal pada domain frekuensi. TF telah dikembangkan di bidang aljabar quaternion yang disebut Transformasi Fourier Quaternion (TFQ) Penelitian ini bertujuan untuk merumuskan definisi dan sifat-sifat Transformasi Fourier Fraksional Quaternion (TFFQ) sisi kiri dan definisi serta teorema konvolusinya. Pertama, hasil penelitian menunjukkan TFFQ sisi kiri dapat dibangun definisi dan beberapa sifatnya seperti linearitas, translasi, modulasi dan skala. Kedua, hasil penelitian ini menunjukkan teorema konvolusi untuk TFFQ sisi kiri dan juga TFFQ sisi kiri dari konjugat dan translasi konvolusi.

Kata Kunci : : Aljabar Quaternion, Teorema Konvolusi, Transformasi Fourier, Transformasi Fraksional Fourier Quaternion. 


\section{PENDAHULUAN}

Transformasi Fourier (TF) adalah salah satu metode matematika yang memiliki banyak manfaat, diantaranya digunakan untuk menganalisa dan melakukan dekomposisi sinyal-sinyal dalam domain frekuensi (Saribulut et al., 2013). Transformasi Fourier dan ilmu yang terkait berkembang sangat pesat karena kegunaannya di berbagai bidang sains dan teknik. Transformasi Fourier Fraksional (TFF) merupakan generalisasi dari TF yang telah banyak diterapkan dalam pengolahan citra dan sinyal (Yetik et al., 2003). Salah satu kegunaan TFF khususnya pada pengolahan sinyal yaitu mampu menganalisa frekuensi pada sinyal frekuensi tinggi (Almeida, 1994).

TF juga telah dikembangkan dalam bidang aljabar quaternion yang disebut Transformasi Fourier Quaternion (TFQ). Menurut Ell dalam Pei dkk. (2001), TFQ ini memiliki beberapa tipe yang berbeda yaitu TFQ tipe I (dua sisi), TFQ tipe II (sisi kiri) dan TFQ tipe III (sisi kanan) karena tidak bersifat komutatif pada operasi perkalian bilangan quaternion. TFQ ini diterapkan dalam pengolahan citra warna dalam domain frekuensinya dan juga dalam perbaikan citra (Hitzer, 2007).

Quaternion merupakan bilangan-bilangan kompleks yang diperluas untuk aljabar dimensi empat dan terdiri dari satu bagian riil dan tiga bagian kompleks (Morais et al., 2010). Bilangan quaternion ini diperkenalkan pertama kali oleh William Rowman Hamilton dan telah digunakan dalam berbagai ilmu seperti kimia, fisika, pemrosesan gambar dan sinyal, komputer grafik serta kompressi data (Irwan, 2015).

Penggunaan bilangan quaternion juga diterapkan dalam TFF yang disebut Transformasi Fourier Fraksional Quaternion (TFFQ). TFFQ dua sisi diperkenalkan pertama kali pada tahun 2008 (Guanlei et al., 2008). Kemudian sifat-sifat konvolusi dari TFFQ dua sisi tersebut dirumuskan pertama kali pada tahun 2016 (Sharma and Deshmukh, 2016). Selain TFFQ dua sisi, definisi TFFQ sisi kanan juga telah dibangun berdasarkan konsep TFQ tipe III (sisi kanan) pada tahun 2013 (Wei and Li, 2013). Pada tahun 2014, teorema sifat-sifat darn konvolusi TFFQ sisi kanan juga telah dirumuskan (Sangkala N, 2014)

\section{METODE PENELITIAN}

Penelitian ini bertempat di Jurusan Matematika FMIPA Universitas Tadulako. Rancangan penelitian ini berbentuk penelitian kualitatif dengan melakukan studi kepustakaan dengan mengumpulkan dan mengkaji materi-materi yang berkaitan dengan aljabar aljabar quaternion, TF, TFF, TFQ dan TFFQ.

Penelitian ini dilakukan dengan melalui tahapan pertama yaitu merumuskan definisi TFFQ sisi kiri. Setelah definisinya dibangun, maka sifat-sifat TFFQ sisi kiri mulai dirumuskan dengan menggunakan definisi TFFQ sisi kiri. Kemudian tahap selanjutnya merumuskan definisi konvolusi pada TFFQ sisi kiri. Setelah definisi konvolusinya dibangun, maka TFFQ sisi kiri dari konvolusi dua 
fungsi quaternion (teorema konvolusi) dapat dirumuskan. Kemudian tahapan selanjutnya merumuskan teorema TFFQ sisi kiri dari sifat-sifat konvolusinya.

\section{HASIL DAN PEMBAHASAN}

Transformasi Fourier Fraksional Quaternion (TFFQ) merupakan generalisasi dari TFF pada bilangan quaternion. Definisi TFFQ sisi kiri yang dibangun pada penelitian ini adalah sebagai berikut.

\section{Definisi 1 (TFFQ Sisi Kiri)}

Misalkan $f \in L^{1}\left(\mathbb{R}^{2} ; \mathbb{H}\right)$ adalah fungsi quaternion dua peubah dengan $f(\boldsymbol{x})=f_{0}(\boldsymbol{x})+\boldsymbol{i} f_{1}(\boldsymbol{x})+$ $\boldsymbol{j} f_{2}(\boldsymbol{x})+\boldsymbol{k} f_{3}(\boldsymbol{x})$ dimana $f_{0}, f_{1}, f_{2}, f_{3} \in \mathbb{R}$ dan $\mu$ adalah sebarang bilangan imaginer yang memenuhi $\mu^{2}=$ -1 . Maka TFFQ sisi kiri didefinisikan sebagai

$$
F_{\mu}^{\alpha, \beta}\{f(\boldsymbol{x})\}(\boldsymbol{\omega})=\int_{\mathbb{R}^{2}} K_{\mu}^{\alpha}\left(x_{1}, \omega_{1}\right) K_{\mu}^{\beta}\left(x_{2}, \omega_{2}\right) f(\boldsymbol{x}) \boldsymbol{d} \boldsymbol{x}
$$

dimana $\boldsymbol{d} \boldsymbol{x}=d x_{1} d x_{2}$ dan $\alpha, \beta=\left[0, \frac{\pi}{2}\right]$ serta kernel dari TFFQ sisi kiri untuk persamaan di atas secara berurutan adalah sebagai berikut

$$
K_{\mu}^{\alpha}\left(x_{1}, \omega_{1}\right)=\sqrt{\frac{1-\mu \cot \alpha}{2 \pi}} e^{\mu\left(\frac{x_{1}^{2}+\omega_{1}^{2}}{2 \tan \alpha}-\frac{x_{1} \omega_{1}}{\sin \alpha}\right)} \text { dan } K_{\mu}^{\beta}\left(x_{2}, \omega_{2}\right)=\sqrt{\frac{1-\mu \cot \beta}{2 \pi}} e^{\mu\left(\frac{x_{2}^{2}+\omega_{2}^{2}}{2 \tan \beta}-\frac{x_{2} \omega_{2}}{\sin \beta}\right)}
$$

Berikut ini akan disajikan beberapa sifat-sifat TFFQ sisi kiri.

\section{a. Teorema 1 (Sifat Linearitas)}

Misalkan $f(\boldsymbol{x})$ dan $g(\boldsymbol{x})$ merupakan dua fungsi bernilai quaternion dengan $f, g \in$ $L^{1}\left(\mathbb{R}^{2} ; \mathbb{H}\right)$ dan $a$ dan $b$ adalah sebarang konstanta riil, maka

$$
F_{\mu}^{\alpha, \beta}\{a f(\boldsymbol{x})+b g(\boldsymbol{x})\}(\boldsymbol{\omega})=a F_{\mu}^{\alpha, \beta}\{f(\boldsymbol{x})\}(\boldsymbol{\omega})+b F_{\mu}^{\alpha, \beta}\{g(\boldsymbol{x})\}(\boldsymbol{\omega})
$$

Bukti :

Dari definisi TFFQ sisi kiri pada persamaan (1), diperoleh

$$
\begin{aligned}
& F_{\mu}^{\alpha, \beta}\{a f(\boldsymbol{x})+b g(\boldsymbol{x})\}(\boldsymbol{\omega}) \\
& =\int_{\mathbb{R}^{2}} K_{\mu}^{\alpha}\left(x_{1}, \omega_{1}\right) K_{\mu}^{\beta}\left(x_{2}, \omega_{2}\right)(a f(\boldsymbol{x})+b g(\boldsymbol{x})) \boldsymbol{d} \boldsymbol{x} \\
& =a \int_{\mathbb{R}^{2}} K_{\mu}^{\alpha}\left(x_{1}, \omega_{1}\right) K_{\mu}^{\beta}\left(x_{2}, \omega_{2}\right)(f(\boldsymbol{x})) \boldsymbol{d} \boldsymbol{x}+b \int_{\mathbb{R}^{2}} K_{\mu}^{\alpha}\left(x_{1}, \omega_{1}\right) K_{\mu}^{\beta}\left(x_{2}, \omega_{2}\right)(g(\boldsymbol{x})) \boldsymbol{d} \boldsymbol{x} \\
& =a F_{\mu}^{\alpha, \beta}\{f(\boldsymbol{x})\}(\boldsymbol{\omega})+b F_{\mu}^{\alpha, \beta}\{g(\boldsymbol{x})\}(\boldsymbol{\omega})
\end{aligned}
$$

Dengan demikian terbukti bahwa TFFQ sisi kiri memenuhi sifat linearitas.

\section{b. Teorema 2 (Sifat Translasi)}

Misalkan $f \in L^{1}\left(\mathbb{R}^{2} ; \mathbb{H}\right)$ dan translasi dari fungsi $f$ didefinisikan sebagai $\tau_{x_{0}} f(\boldsymbol{x})=$ $f\left(x_{1}-x_{01}, x_{2}-x_{02}\right)$ dimana $x_{01}, x_{02} \in \mathbb{R}$, maka

$$
\begin{aligned}
F_{\mu}^{\alpha, \beta}\left\{\tau_{x_{0}} f(\boldsymbol{x})\right\}(\boldsymbol{\omega})= & e^{\mu\left(\omega_{1} x_{01} \sin \alpha+\omega_{2} x_{02}(\sin \beta)\right)} e^{-\frac{1}{2} \mu\left(x_{01}{ }^{2} \cos \alpha \sin \alpha+x_{02}{ }^{2} \cos \beta \sin \beta\right)} \\
& \cdot F_{\mu}^{\alpha, \beta}\{f\}\left(\omega_{1}-x_{1} \cos \alpha, \omega_{2}-x_{2} \cos \beta\right)
\end{aligned}
$$

Bukti :

Dari definisi TFFQ sisi kiri pada persamaan (1) diketahui $F_{\mu}^{\alpha, \beta}\left\{\tau_{x_{0}} f(\boldsymbol{x})\right\}(\boldsymbol{\omega})$ 


$$
=\int_{\mathbb{R}^{2}} \sqrt{\frac{1-\mu \cot \alpha}{2 \pi}} e^{\mu\left(\frac{x_{1}^{2}+\omega_{1}^{2}}{2 \tan \alpha}-\frac{x_{1} \omega_{1}}{\sin \alpha}\right)} \sqrt{\frac{1-\mu \cot \beta}{2 \pi}} e^{\mu\left(\frac{x_{2}^{2}+\omega_{2}^{2}}{2 \tan \beta}-\frac{x_{2} \omega_{2}}{\sin \beta}\right)} f\left(x_{1}-x_{01}, x_{2}-x_{02}\right) \boldsymbol{d} \boldsymbol{x}
$$

Misalkan $\boldsymbol{t}=\left(t_{1}, t_{2}\right)$ dengan $t_{1}=x_{1}-x_{01}$ dan $t_{2}=x_{2}-x_{02}$. Selanjutnya lakukan manipulasi aljabar sehingga menjadi

$$
\begin{aligned}
& F_{\mu}^{\alpha, \beta}\left\{\tau_{x_{0}} f(\boldsymbol{x})\right\}(\boldsymbol{\omega}) \\
& =\int_{\mathbb{R}^{2}} \sqrt{\frac{1-\mu \cot \alpha}{2 \pi}} e^{\frac{1}{2} \mu\left(\frac{\cos \alpha}{\sin \alpha} t_{1}{ }^{2}-\frac{2}{\sin \alpha} t_{1}\left(\omega_{1}-\cos \alpha x_{01}\right)+\frac{\cos \alpha}{\sin \alpha}\left(\omega_{1}-\cos \alpha x_{0} 1\right)^{2}\right)} e^{\mu \omega_{1} x_{01}\left(\frac{\cos ^{2} \alpha}{\sin \alpha}-\frac{1}{\sin \alpha}\right)} \\
& \cdot e^{-\frac{1}{2} \mu x_{01}{ }^{2} \cos \alpha\left(\frac{\cos ^{2} \alpha}{\sin \alpha}-\frac{1}{\sin \alpha}\right)} \sqrt{\frac{1-\mu \cot \beta}{2 \pi}} e^{\frac{1}{2} \mu\left(\frac{\cos \beta}{\sin \beta} t_{2}{ }^{2}-\frac{2}{\sin \beta} t_{2}\left(\omega_{2}-\cos \beta x_{02}\right)+\frac{\cos \beta}{\sin \beta}\left(\omega_{2}-\cos \beta x_{02}\right)^{2}\right)} \\
& \cdot e^{\mu \omega_{2} x_{02}\left(\frac{\cos ^{2} \beta}{\sin \beta}-\frac{1}{\sin \beta}\right)} e^{-\frac{1}{2} \mu x_{02}{ }^{2} \cos \beta\left(\frac{\cos ^{2} \beta}{\sin \beta}-\frac{1}{\sin \beta}\right)} f(\boldsymbol{t}) \boldsymbol{d} \boldsymbol{t}
\end{aligned}
$$

Jika diketahui bahwa $\cos ^{2} \alpha+\sin ^{2} \alpha=1$ dan $\cos ^{2} \beta+\sin ^{2} \beta=1$. Maka diperoleh

$$
\begin{aligned}
& F_{\mu}^{\alpha, \beta}\left\{\tau_{x_{0}} f(\boldsymbol{x})\right\}(\boldsymbol{\omega}) \\
& =\int_{\mathbb{R}^{2}} e^{\mu\left(\omega_{1} x_{01} \sin \alpha+\omega_{2} x_{02}(\sin \beta)\right)} e^{-\frac{1}{2} \mu\left(x_{01}{ }^{2} \cos \alpha \sin \alpha+x_{02}{ }^{2} \cos \beta \sin \beta\right)} \\
& \cdot \sqrt{\frac{1-\mu \cot \alpha}{2 \pi}} e^{\frac{1}{2} \mu\left(\frac{\cos \alpha}{\sin \alpha} t_{1}{ }^{2}-\frac{2}{\sin \alpha} t_{1}\left(\omega_{1}-\cos \alpha x_{01}\right)+\frac{\cos \alpha}{\sin \alpha}\left(\omega_{1}-\cos \alpha x_{01}\right)^{2}\right)} \sqrt{\frac{1-\mu \cot \beta}{2 \pi}} \\
& \cdot e^{\frac{1}{2} \mu\left(\frac{\cos \beta}{\sin \beta} t_{2}{ }^{2}-\frac{2}{\sin \beta} t_{2}\left(\omega_{2}-\cos \beta x_{02}\right)+\frac{\cos \beta}{\sin \beta}\left(\omega_{2}-\cos \beta x_{02}\right)^{2}\right)} f(\boldsymbol{t}) \boldsymbol{d} \boldsymbol{t}
\end{aligned}
$$

Berdasarkan definisi TFFQ sisi kiri, maka diperoleh

$$
\begin{aligned}
F_{\mu}^{\alpha, \beta}\left\{\tau_{x_{0}} f(\boldsymbol{x})\right\}(\boldsymbol{\omega})= & e^{\mu\left(\omega_{1} x_{01} \sin \alpha+\omega_{2} x_{02} \sin \beta\right)} e^{-\frac{1}{2} \mu\left(x_{01}{ }^{2} \cos \alpha \sin \alpha+x_{02}{ }^{2} \cos \beta \sin \beta\right)} \\
& \cdot F_{\mu}^{\alpha, \beta}\{f(\boldsymbol{x})\}\left(\omega_{1}-x_{1} \cos \alpha, \omega_{2}-x_{2} \cos \beta\right)
\end{aligned}
$$

Dengan demikian terbukti bahwa TFFQ sisi kiri memenuhi sifat translasi.

\section{c. Teorema 3 (Sifat Modulasi)}

Misalkan $f \in L^{1}\left(\mathbb{R}^{2} ; \mathbb{H}\right)$ dan modulasi dari fungsi $f$ dinyatakan sebagai $f(\boldsymbol{x}) e^{\mu \omega_{1} x_{1}} e^{\mu \omega_{2} x_{2}}$, maka sifat modulasi dari TFFQ sisi kiri yaitu $F_{\mu}^{\alpha, \beta}\left\{f(\boldsymbol{x}) e^{\mu \omega_{1} x_{1}} e^{\mu \omega_{2} x_{2}}\right\}(\boldsymbol{\omega})$

$$
\begin{aligned}
= & e^{\mu\left(\cos \alpha\left(\omega_{1}{ }^{2}-\sin \alpha \omega_{1}{ }^{2}\right)+\cos \beta\left(\omega_{2}{ }^{2}-\sin \beta \omega_{2}{ }^{2}\right)\right)} e^{\frac{1}{2} \mu\left(\cos \alpha \sin \alpha \omega_{1}{ }^{2}+\cos \beta \sin \beta \omega_{2}{ }^{2}\right)} \\
& \cdot F_{\mu}^{\alpha, \beta}\{f\}\left(\omega_{1}-\sin \alpha \omega_{1}, \omega_{2}-\sin \beta \omega_{2}\right)
\end{aligned}
$$

Bukti :

Dari definisi TFFQ sisi kiri pada persamaan (1) diperoleh

$$
\begin{aligned}
& F_{\mu}^{\alpha, \beta}\left\{f(\boldsymbol{x}) e^{\mu \omega_{1} x_{1}} e^{\mu \omega_{2} x_{2}}\right\}(\boldsymbol{\omega}) \\
& =\int_{\mathbb{R}^{2}} \sqrt{\frac{1-\mu \cot \alpha}{2 \pi}} e^{\frac{1}{2} \mu\left(\frac{\cos \alpha}{\sin \alpha} x_{1}^{2}-\frac{2 x_{1}\left(\omega_{1}-\sin \alpha\right)}{\sin \alpha}+\frac{\cos \alpha}{\sin \alpha} \omega_{1}^{2}\right)} \sqrt{\frac{1-\mu \cot \beta}{2 \pi}} \\
& \cdot e^{\frac{1}{2} \mu\left(\frac{\cos \beta}{\sin \beta} x_{02}{ }^{2}-\frac{2 x_{02}\left(\omega_{2}-\sin \beta\right)}{\sin \beta}+\frac{\cos \beta}{\sin \beta} \omega_{2}^{2}\right)} f(\boldsymbol{x}) \boldsymbol{d} \boldsymbol{x}
\end{aligned}
$$

Selanjutnya bentuk $e^{\frac{1}{2} \mu\left(\frac{\cos \alpha}{\sin \alpha} \omega_{1}^{2}\right)}$ dan $e^{\frac{1}{2} \mu\left(\frac{\cos \beta}{\sin \beta} \omega_{2}^{2}\right)}$ pada persamaan di atas ditulis dalam bentuk $e^{\frac{1}{2} \mu\left(\frac{\cos \alpha}{\sin \alpha}\left(\omega_{1}-\sin \alpha \omega_{1}+\sin \alpha \omega_{1}\right)^{2}\right)}$ dan $e^{\frac{1}{2} \mu\left(\frac{\cos \beta}{\sin \beta}\left(\omega_{2}-\sin \beta \omega_{2}+\sin \beta \omega_{2}\right)^{2}\right)}$, diperoleh 


$$
\begin{aligned}
& F_{\mu}^{\alpha, \beta}\left\{f(\boldsymbol{x}) e^{\mu \omega_{1} x_{1}} e^{\mu \omega_{2} x_{2}}\right\}(\boldsymbol{\omega}) \\
& =\int_{\mathbb{R}^{2}} \sqrt{\frac{1-\mu \cot \alpha}{2 \pi}} e^{\frac{1}{2} \mu\left(\frac{\cos \alpha}{\sin \alpha} x_{1}^{2}-\frac{2 x_{1}\left(\omega_{1}-\sin \alpha\right)}{\sin \alpha}+\frac{\cos \alpha}{\sin \alpha}\left(\left(\omega_{1}-\sin \alpha \omega_{1}\right)+\sin \alpha \omega_{1}\right)^{2}\right)} \sqrt{\frac{1-\mu \cot \beta}{2 \pi}} \\
& \cdot e^{\frac{1}{2} \mu\left(\frac{\cos \beta}{\sin \beta} x_{2}^{2}-\frac{2 x_{2}\left(\omega_{2}-\sin \beta\right)}{\sin \beta}+\frac{\cos \beta}{\sin \beta}\left(\left(\omega_{2}-\sin \beta \omega_{2}\right)+\sin \beta \omega_{2}\right)^{2}\right)} f(\boldsymbol{x}) \boldsymbol{d} \boldsymbol{x}
\end{aligned}
$$

Setelah melalui proses manipulasi aljabar, dengan mudah dapat diperoleh

$$
\begin{gathered}
F_{\mu}^{\alpha, \beta}\left\{f(\boldsymbol{x}) e^{\mu \omega_{1} x_{1}} e^{\mu \omega_{2} x_{2}}\right\}(\boldsymbol{\omega}) \\
=\int_{\mathbb{R}^{2}} e^{\mu\left(\cos \alpha\left(\omega_{1}^{2}-\sin \alpha \omega_{1}^{2}\right)+\cos \beta\left(\omega_{2}^{2}-\sin \beta \omega_{2}^{2}\right)\right)} e^{\frac{1}{2} \mu\left(\cos \alpha \sin \alpha \omega_{1}^{2}+\cos \beta \sin \beta \omega_{2}^{2}\right)} \\
\cdot \sqrt{\frac{1-\mu \cot \alpha}{2 \pi}} e^{\frac{1}{2} \mu\left(\frac{\cos \alpha}{\sin \alpha} x_{1}^{2}-\frac{2 x_{1}\left(\omega_{1}-\sin \alpha\right)}{\sin \alpha}+\frac{\cos \alpha}{\sin \alpha}\left(\left(\omega_{1}-\sin \alpha \omega_{1}\right)^{2}\right)\right)} \sqrt{\frac{1-\mu \cot \beta}{2 \pi}} \\
\cdot e^{\frac{1}{2} \mu\left(\frac{\cos \beta}{\sin \beta} x_{2}^{2}-\frac{2 x_{2}\left(\omega_{2}-\sin \beta\right)}{\sin \beta}+\frac{\cos \beta}{\sin \beta}\left(\left(\omega_{2}-\sin \beta \omega_{2}\right)^{2}\right)\right)} f(\boldsymbol{x}) \boldsymbol{d} \boldsymbol{x}
\end{gathered}
$$

Berdasarkan definisi TFFQ sisi kiri, maka diperoleh

$$
\begin{aligned}
& F_{\mu}^{\alpha, \beta}\left\{f(\boldsymbol{x}) e^{\mu \omega_{1} x_{1}} e^{\mu \omega_{2} x_{2}}\right\}(\boldsymbol{\omega}) \\
& =e^{\mu\left(\cos \alpha\left(\omega_{1}{ }^{2}-\sin \alpha \omega_{1}{ }^{2}\right)+\cos \beta\left(\omega_{2}{ }^{2}-\sin \beta \omega_{2}{ }^{2}\right)\right)} e^{\frac{1}{2} \mu\left(\cos \alpha \sin \alpha \omega_{1}{ }^{2}+\cos \beta \sin \beta \omega_{2}{ }^{2}\right)} \\
& \quad \cdot F_{\mu}^{\alpha, \beta}\{f(\boldsymbol{x})\}\left(\omega_{1}-\sin \alpha \omega_{1}, \omega_{2}-\sin \beta \omega_{2}\right)
\end{aligned}
$$

Dengan demikian terbukti bahwa TFFQ sisi kiri memenuhi sifat modulasi.

\section{d. Teorema 4 (Sifat Skala)}

Misalkan $f \in L^{1}\left(\mathbb{R}^{2} ; \mathbb{H}\right)$ dan skala dari fungsi $f$ didefinisikan sebagai $f_{m}(\boldsymbol{x})=$ $f\left(m x_{1}, m x_{2}\right)$, dengan $m \in \mathbb{R}$ adalah konstanta tidak nol, maka

a. Untuk kasus $m>0$

$$
\begin{aligned}
& F_{\mu}^{\alpha, \beta}\left\{f_{m}(\boldsymbol{x})\right\}(\boldsymbol{\omega}) \\
& =\sqrt{\frac{1-\mu \cot \alpha}{m^{2}-\mu \cot \alpha}} \sqrt{\frac{1-\mu \cot \beta}{m^{2}-\mu \cot \beta}} e^{\frac{1}{2} \mu\left(\omega_{1}^{2} \cot \alpha\right)\left(1-\frac{\csc ^{2} \alpha}{m^{4} \csc ^{2} \theta_{1}}\right)} e^{\frac{1}{2} \mu\left(\omega_{2}^{2} \cot \beta\right)\left(1-\frac{\csc ^{2} \beta}{m^{4} \csc ^{2} \theta_{2}}\right)} \\
& \cdot F_{\mu}^{\theta_{1}, \theta_{2}}\{f(m \boldsymbol{x})\}\left(\omega_{1} \frac{\csc \alpha}{m \csc \theta_{1}}, \omega_{1} \frac{\csc \beta}{m \csc \theta_{2}}\right)
\end{aligned}
$$

b. Untuk kasus $m<0$

$$
\begin{aligned}
& F_{\mu}^{\alpha, \beta}\left\{f_{m}(\boldsymbol{x})\right\}(\boldsymbol{\omega}) \\
& =\sqrt{\frac{1-\mu \cot \alpha}{m^{2}-\mu \cot \alpha}} \sqrt{\frac{1-\mu \cot \beta}{m^{2}-\mu \cot \beta}} e^{\frac{1}{2} \mu\left(4 t_{1} \omega_{1} \frac{\csc \alpha}{m \csc \theta_{1}}+4 t_{2} \omega_{2} \frac{\csc \beta}{m \csc \theta_{2}}\right)} e^{\frac{1}{2} \mu\left(\omega_{1}^{2} \cot \alpha\right)\left(1-\frac{\csc ^{2} \alpha}{m^{4} \csc ^{2} \theta_{1}}\right)} \\
& \text { - } e^{\frac{1}{2} \mu\left(\omega_{2}^{2} \cot \beta\right)\left(1-\frac{\csc ^{2} \beta}{m^{4} \csc ^{2} \theta_{2}}\right)} F_{\mu}^{\theta_{1}, \theta_{2}}\{f(m x)\}\left(\omega_{1} \frac{\csc \alpha}{m \csc \theta_{1}}, \omega_{2} \frac{\csc \beta}{m \csc \theta_{2}}\right) \\
& \text { dimana } \theta_{1}=\cot ^{-1}\left(\frac{\cot \alpha}{m^{2}}\right) \operatorname{dan} \theta_{2}=\cot ^{-1}\left(\frac{\cot \beta}{m^{2}}\right)
\end{aligned}
$$

Bukti :

Dari definisi TFFQ sisi kiri pada persamaan (1), diketahui

$$
\begin{aligned}
= & \int_{\mathbb{R}^{2}} \sqrt{\frac{1-\mu \cot \alpha}{2 \pi}} e^{\frac{1}{2} \mu\left(\frac{\cos \alpha}{\sin \alpha} x_{1}^{2}-\frac{2 x_{1} \omega_{1}}{\sin \alpha}+\frac{\cos \alpha}{\sin \alpha} \omega_{1}^{2}\right)} \sqrt{\frac{1-\mu \cot \beta}{2 \pi}} \\
& \cdot e^{\frac{1}{2} \mu\left(\frac{\cos \beta}{\sin \beta} x_{2}^{2}-\frac{2 x_{2} \omega_{2}}{\sin \beta}+\frac{\cos \beta}{\sin \beta} \omega_{2}^{2}\right)} f(\boldsymbol{x}) \boldsymbol{d} \boldsymbol{x}
\end{aligned}
$$

Misalkan : $\boldsymbol{t}=\left(t_{1}, t_{2}\right)=\left(m x_{1}, m x_{2}\right)$, dari pemisalan ini diperoleh $x_{1}=\frac{t_{1}}{m}$ dan $x_{2}=\frac{t_{2}}{m}$. 
Berdasarkan nilai skala $(m)$, maka pembuktian ini dibagi menjadi 2 kasus yaitu untuk $m>0$ dan $m<0$ sebagai berikut.

a. Kasus 1 (untuk $m>0$ )

$$
\begin{aligned}
& F_{\mu}^{\alpha, \beta}\left\{f\left(m x_{1}, m x_{2}\right)\right\}(\boldsymbol{\omega}) \\
& =\frac{1}{m^{2}} \int_{\mathbb{R}^{2}} \sqrt{\frac{1-\mu \cot \alpha}{2 \pi}} e^{\frac{1}{2} \mu\left(\frac{\cos \alpha}{\sin \alpha}\left(\frac{t_{1}}{m}\right)^{2}-\frac{2\left(\frac{t_{1}}{m}\right) \omega_{1}}{\sin \alpha}+\frac{\cos \alpha}{\sin \alpha} \omega_{1}^{2}\right)} \sqrt{\frac{1-\mu \cot \beta}{2 \pi}} \\
& \cdot e^{\frac{1}{2} \mu\left(\frac{\cos \beta}{\sin \beta}\left(\frac{t_{2}}{m}\right)^{2}-\frac{2\left(\frac{t_{2}}{m}\right) \omega_{2}}{\sin \beta}+\frac{\cos \beta}{\sin \beta} \omega_{2}^{2}\right)} f(\boldsymbol{t}) \boldsymbol{d} \boldsymbol{t}
\end{aligned}
$$

Misalkan $\theta_{1}=\cot ^{-1}\left(\frac{\cot \alpha}{m^{2}}\right)$ dan $\theta_{2}=\cot ^{-1}\left(\frac{\cot \beta}{m^{2}}\right)$. Setelah melalui proses manipulasi aljabar, dengan mudah diperoleh

$$
\begin{aligned}
& F_{\mu}^{\alpha, \beta}\left\{f\left(m x_{1}, m x_{2}\right)\right\}(\boldsymbol{\omega}) \\
& =\int_{\mathbb{R}^{2}} \sqrt{\frac{1-\mu \cot \alpha}{m^{2}-\mu \cot \alpha}} e^{\frac{1}{2} \mu\left(\left(\omega_{1}\right)^{2} \cot \alpha\right)\left(1-\frac{\csc ^{2} \alpha}{m^{4} \csc ^{2} \theta_{1}}\right)} \sqrt{\frac{1-\mu \cot \beta}{m^{2}-\mu \cot \beta}} e^{\frac{1}{2} \mu\left(\left(\omega_{2}\right)^{2} \cot \beta\right)\left(1-\frac{\csc ^{2} \beta}{m^{4} \csc ^{2} \theta_{2}}\right)} \\
& \cdot \sqrt{\frac{1-\mu \cot \theta_{1}}{2 \pi}} e^{\frac{1}{2} \mu\left(\left(t_{1}\right)^{2} \cot \theta_{1}-2 t_{1} \omega_{1} \frac{\csc \alpha}{m \csc \theta_{1}} \csc \theta_{1}+\left(\omega_{1} \frac{\csc \alpha}{m \csc \theta_{1}}\right)^{2} \cot \theta_{1}\right) \sqrt{\frac{1-\mu \cot \theta_{2}}{2 \pi}}} \\
& \cdot e^{\frac{1}{2} \mu\left(\left(t_{2}\right)^{2} \cot \theta_{2}-2 t_{2} \omega_{2} \frac{\csc \beta}{m \csc \theta_{2}} \csc \theta_{2}+\left(\omega_{2} \frac{\csc \beta}{m \csc \theta_{2}}\right)^{2} \cot \theta_{2}\right)} f\left(m x_{1}, m x_{2}\right) d t
\end{aligned}
$$

Misalkan $\left(m x_{1}, m x_{2}\right)=m \boldsymbol{x}$. Berdasarkan definisi TFFQ sisi kiri, maka diperoleh

$$
\begin{aligned}
& F_{\mu}^{\alpha, \beta}\left\{f_{m}(\boldsymbol{x})\right\}(\boldsymbol{\omega}) \\
& =\sqrt{\frac{1-\mu \cot \alpha}{m^{2}-\mu \cot \alpha}} \sqrt{\frac{1-\mu \cot \beta}{m^{2}-\mu \cot \beta}} e^{\frac{1}{2} \mu\left(\omega_{1}{ }^{2} \cot \alpha\right)\left(1-\frac{\csc ^{2} \alpha}{m^{4} \csc ^{2} \theta_{1}}\right)} e^{\frac{1}{2} \mu\left(\omega_{2}{ }^{2} \cot \beta\right)\left(1-\frac{\csc ^{2} \beta}{m^{4} \csc ^{2} \theta_{2}}\right)} \\
& \text { - } F_{\mu}^{\theta_{1}, \theta_{2}}\{f(m \boldsymbol{x})\}\left(\omega_{1} \frac{\csc \alpha}{m \csc \theta_{1}}, \omega_{1} \frac{\csc \beta}{m \csc \theta_{2}}\right)
\end{aligned}
$$

Dengan demikian terbukti bahwa TFFQ sisi kiri memenuhi sifat skala untuk $m>0$.

b. Kasus 2 (untuk $m<0$ )

$$
\begin{aligned}
& F_{\mu}^{\alpha, \beta}\left\{f\left(m x_{1}, m x_{2}\right)\right\}(\boldsymbol{\omega}) \\
& =\frac{1}{m^{2}} \int_{\mathbb{R}^{2}} \sqrt{\frac{1-\mu \cot \alpha}{2 \pi}} e^{\frac{1}{2} \mu\left(\frac{\cos \alpha}{\sin \alpha}\left(\frac{t_{1}}{m}\right)^{2}-\left(\frac{-2\left(\frac{t_{1}}{m}\right) \omega_{1}}{\sin \alpha}\right)+\frac{\cos \alpha}{\sin \alpha} \omega_{1}^{2}\right)} \sqrt{\frac{1-\mu \cot \beta}{2 \pi}} \\
& \cdot e^{\frac{1}{2} \mu\left(\frac{\cos \beta}{\sin \beta}\left(\frac{t_{2}}{m}\right)^{2}-\left(\frac{-2\left(\frac{t_{2}}{m}\right) \omega_{2}}{\sin \beta}\right)+\frac{\cos \beta}{\sin \beta} \omega_{2}^{2}\right)} f(\boldsymbol{t}) \boldsymbol{d} \boldsymbol{t}
\end{aligned}
$$

Misalkan $\theta_{1}=\cot ^{-1}\left(\frac{\cot \alpha}{m^{2}}\right)$ dan $\theta_{2}=\cot ^{-1}\left(\frac{\cot \beta}{m^{2}}\right)$. Setelah melalui proses manipulasi aljabar, dengan mudah diperoleh

$$
\begin{aligned}
& F_{\mu}^{\alpha, \beta}\left\{f_{m}(\boldsymbol{x})\right\}(\boldsymbol{\omega}) \\
& =\int_{\mathbb{R}^{2}} \sqrt{\frac{1-\mu \cot \alpha}{m^{2}-\mu \cot \alpha}} e^{\frac{1}{2} \mu\left(\left(\omega_{1}\right)^{2} \cot \alpha\right)\left(1-\frac{\csc ^{2} \alpha}{m^{4} \csc ^{2} \theta_{1}}\right)} \sqrt{\frac{1-\mu \cot \beta}{m^{2}-\mu \cot \beta}} e^{\frac{1}{2} \mu\left(\left(\omega_{2}\right)^{2} \cot \beta\right)\left(1-\frac{\csc ^{2} \beta}{m^{4} \csc ^{2} \theta_{2}}\right)} \\
& \cdot e^{\frac{1}{2} \mu\left(4 t_{1} \omega_{1} \frac{\csc \alpha}{m \csc \theta_{1}}+4 t_{2} \omega_{2} \frac{\csc \beta}{m \csc \theta_{2}}\right) e^{\frac{1}{2} \mu\left(\left(t_{1}\right)^{2} \cot \theta_{1}-2 t_{1} \omega_{1} \frac{\csc \alpha}{m \csc \theta_{1}} \csc \theta_{1}+\left(\omega_{1} \frac{\csc \alpha}{m \csc \theta_{1}}\right)^{2} \cot \theta_{1}\right)}} \\
& \cdot \sqrt{\frac{1-\mu \cot \theta_{1}}{2 \pi}} e^{\frac{1}{2} \mu\left(\left(t_{2}\right)^{2} \cot \theta_{2}-2 t_{2} \omega_{2} \frac{\csc \beta}{m \csc \theta_{2}} \csc \theta_{2}+\left(\omega_{2} \frac{\csc \beta}{m \csc \theta_{2}}\right)^{2} \cot \theta_{2}\right)} \sqrt{\frac{1-\mu \cot \theta_{2}}{2 \pi}} f(m \boldsymbol{x}) \boldsymbol{d t}
\end{aligned}
$$


Berdasarkan definisi TFFQ sisi kiri, maka diperoleh

$$
\begin{aligned}
& F_{\mu}^{\alpha, \beta}\left\{f_{m}(\boldsymbol{x})\right\}(\boldsymbol{\omega}) \\
& =\sqrt{\frac{1-\mu \cot \alpha}{m^{2}-\mu \cot \alpha}} \sqrt{\frac{1-\mu \cot \beta}{m^{2}-\mu \cot \beta}} e^{\frac{1}{2} \mu\left(4 t_{1} \omega_{1} \frac{\csc \alpha}{m \csc \theta_{1}}+4 t_{2} \omega_{2} \frac{\csc \beta}{m \csc \theta_{2}}\right)} e^{\frac{1}{2} \mu\left(\omega_{1}^{2} \cot \alpha\right)\left(1-\frac{\csc ^{2} \alpha}{m^{4} \csc ^{2} \theta_{1}}\right)} \\
& \cdot e^{\frac{1}{2} \mu\left(\omega_{2}^{2} \cot \beta\right)\left(1-\frac{\csc ^{2} \beta}{m^{4} \csc ^{2} \theta_{2}}\right)} F_{\mu}^{\theta_{1}, \theta_{2}}\{f(m \boldsymbol{x})\}\left(\omega_{1} \frac{\csc \alpha}{m \csc \theta_{1}}, \omega_{2} \frac{\csc \beta}{m \csc \theta_{2}}\right)
\end{aligned}
$$

Dengan demikian terbukti bahwa TFFQ sisi kiri memenuhi sifat skala untuk $m<0$

Berikut ini definisi konvolusi pada TFFQ sisi kiri, teorema konvolusi pada TFFQ sisi kiri dan TFFQ sisi kiri dari sifat-sifat konvolusinya.

\section{Definisi 2 (Konvolusi padaTFFQ Sisi Kiri)}

Misalkan $f, g \in L^{1}\left(\mathbb{R}^{2} ; \mathbb{H}\right)$ adalah dua fungsi sebarang yang bernilai quaternion, konvolusi TFFQ sisi kiri didefinisikan sebagai

$$
\begin{aligned}
& (f \star g)(\boldsymbol{x})=\int_{\mathbb{R}^{2}} \sqrt{\frac{1-\mu \cot \alpha}{2 \pi}} e^{\frac{\cos \alpha}{\sin \alpha} \mu t_{1}\left(t_{1}-x_{1}\right)} \sqrt{\frac{1-\mu \cot \beta}{2 \pi}} e^{\frac{\cos \beta}{\sin \beta} \mu t_{2}\left(t_{2}-x_{2}\right)} \\
& \quad \cdot f(\boldsymbol{t}) g(\boldsymbol{x}-\boldsymbol{t}) \boldsymbol{d} \boldsymbol{t} \\
& \text { dengan }(\boldsymbol{x}-\boldsymbol{t})=\left(x_{1}-t_{1}, x_{2}-t_{2}\right)
\end{aligned}
$$

\section{a. Teorema 5 (TFFQ sisi kiri dari Konvolusi)}

Misalkan $f(\boldsymbol{x})=f_{0}(\boldsymbol{x})+\boldsymbol{i} f_{1}(\boldsymbol{x})+\boldsymbol{j} f_{2}(\boldsymbol{x})+\boldsymbol{k} f_{3}(\boldsymbol{x})$ dan $g(\boldsymbol{x})=g_{0}(\boldsymbol{x})+\boldsymbol{i} g_{1}(\boldsymbol{x})+\boldsymbol{j} g_{2}(\boldsymbol{x})+$ $\boldsymbol{k} f_{3}(\boldsymbol{x})$ merupakan dua fungsi bernilai quaternion, maka TFFQ sisi kiri dari konvolusi fungsi $f$ dan $g$ dengan $f, g \in L^{1}\left(\mathbb{R}^{2} ; \mathbb{H}\right)$ memenuhi

$$
\begin{aligned}
& F_{\mu}^{\alpha, \beta}\{f \star g\}(\boldsymbol{\omega}) \\
& =e^{-\frac{1}{2} \frac{\cos \alpha}{\sin \alpha} \mu \omega_{1}{ }^{2}} e^{-\frac{1}{2} \frac{\cos \beta}{\sin \beta} \mu \omega_{2}{ }^{2}}\left[F_{\mu}^{\alpha, \beta}\left\{f_{0}(\boldsymbol{t})\right\}(\boldsymbol{\omega}) F_{\mu}^{\alpha, \beta}\{g(\boldsymbol{x}-\boldsymbol{t})\}(\boldsymbol{\omega})\right. \\
& \quad+\boldsymbol{i} F_{\mu}^{\alpha, \beta}\left\{f_{1}(\boldsymbol{t})\right\}(\boldsymbol{\omega}) F_{\mu}^{\alpha, \beta}\{g(\boldsymbol{x}-\boldsymbol{t})\}(\boldsymbol{\omega})+\boldsymbol{j} F_{\mu}^{\alpha, \beta}\left\{f_{2}(\boldsymbol{t})\right\}(\boldsymbol{\omega}) F_{\mu}^{\alpha, \beta}\{g(\boldsymbol{x}-\boldsymbol{t})\}(\boldsymbol{\omega}) \\
& \left.\quad+\boldsymbol{k} F_{\mu}^{\alpha, \beta}\left\{f_{3}(\boldsymbol{t})\right\}(\boldsymbol{\omega}) F_{\mu}^{\alpha, \beta}\{g(\boldsymbol{x}-\boldsymbol{t})\}(\boldsymbol{\omega})\right]
\end{aligned}
$$

Bukti :

Dari definisi TFFQ sisi kiri pada persamaan (1) dan definisi konvolusi pada persamaan (7), diketahui

$$
\begin{aligned}
& F_{\mu}^{\alpha, \beta}\{f \star g\}(\boldsymbol{\omega}) \\
& =\int_{\mathbb{R}^{2}} \int_{\mathbb{R}^{2}} \sqrt{\frac{1-\mu \cot \alpha}{2 \pi}} e^{\frac{1}{2} \mu\left(\frac{\cos \alpha}{\sin \alpha} x_{1}{ }^{2}-\frac{2}{\sin \alpha} x_{1} \omega_{1}+\frac{\cos \alpha}{\sin \alpha} \omega_{1}{ }^{2}\right)} \sqrt{\frac{1-\mu \cot \beta}{2 \pi}} \\
& \cdot e^{\frac{1}{2} \mu\left(\frac{\cos \beta}{\sin \beta} x_{2}{ }^{2}-\frac{2}{\sin \beta} x_{2} \omega_{2}+\frac{\cos \beta}{\sin \beta} \omega_{2}{ }^{2}\right)} \sqrt{\frac{1-\mu \cot \alpha}{2 \pi}} e^{\frac{\cos \alpha}{\sin \alpha} \mu t_{1}\left(t_{1}-x_{1}\right)} \sqrt{\frac{1-\mu \cot \beta}{2 \pi}} \\
& \cdot e^{\frac{\cos \beta}{\sin \beta} \mu t_{2}\left(t_{2}-x_{2}\right)} f(\boldsymbol{t}) g(\boldsymbol{x}-\boldsymbol{t}) \boldsymbol{d} \boldsymbol{d} \boldsymbol{x}
\end{aligned}
$$

Misalkan $\boldsymbol{\varphi}=(\boldsymbol{x}-\boldsymbol{t})$. Setelah melakukan manipulasi aljabar, dengan mudah diperoleh $F_{\mu}^{\alpha, \beta}\{f \star g\}(\omega)$ 


$$
\begin{aligned}
= & \sqrt{\frac{1-\mu \cot \alpha}{2 \pi}} \sqrt{\frac{1-\mu \cot \beta}{2 \pi}} \int_{\mathbb{R}^{2}} e^{\frac{1 \cos \alpha}{2 \sin \alpha} \mu t_{1}{ }^{2}} e^{\frac{1}{2} \mu\left(-\frac{2}{\sin \alpha} t_{1} \omega_{1}\right)} e^{\frac{1 \cos \beta}{2 \sin \beta} \mu t_{2}{ }^{2}} e^{\frac{1}{2} \mu\left(-\frac{2}{\sin \beta} t_{2} \omega_{2}\right)} \\
& \cdot f(\boldsymbol{t}) F_{\mu}^{\alpha, \beta}\{g(\boldsymbol{\varphi})\}(\boldsymbol{\omega}) \boldsymbol{d} \boldsymbol{t}
\end{aligned}
$$

Karena tidak berlaku sifat komutatif pada operasi perkalian bilangan quaternion, maka fungsi $f(\boldsymbol{t})$ harus didekomposisi menjadi $f(\boldsymbol{t})=f_{0}(\boldsymbol{t})+\boldsymbol{i} f_{1}(\boldsymbol{t})+\boldsymbol{j} f_{2}(\boldsymbol{t})+\boldsymbol{k} f_{3}(\boldsymbol{t})$, sehingga diperoleh $F_{\mu}^{\alpha, \beta}\{f \star g\}(\boldsymbol{\omega})$

$$
\begin{aligned}
= & \sqrt{\frac{1-\mu \cot \alpha}{2 \pi}} \sqrt{\frac{1-\mu \cot \beta}{2 \pi}} \int_{\mathbb{R}^{2}} e^{\frac{1 \cos \alpha}{2 \sin \alpha} \mu t_{1}{ }^{2}} e^{\frac{1}{2} \mu\left(-\frac{2}{\sin \alpha} t_{1} \omega_{1}\right)} e^{\frac{1 \cos \beta}{2 \sin \beta} \mu t_{2}{ }^{2}} e^{\frac{1}{2} \mu\left(-\frac{2}{\sin \beta} t_{2} \omega_{2}\right)} \\
& \cdot\left\{F_{\mu}^{\alpha, \beta}\{g(\boldsymbol{\varphi})\}(\boldsymbol{\omega}) f_{0}(\boldsymbol{t})+\boldsymbol{i} F_{\mu}^{\alpha, \beta}\{g(\boldsymbol{\varphi})\}(\boldsymbol{\omega}) f_{1}(\boldsymbol{t})+\boldsymbol{j} F_{\mu}^{\alpha, \beta}\{g(\boldsymbol{\varphi})\}(\boldsymbol{\omega}) f_{2}(\boldsymbol{t})\right. \\
& \left.+\boldsymbol{k} F_{\mu}^{\alpha, \beta}\{g(\boldsymbol{\varphi})\}(\boldsymbol{\omega}) f_{3}(\boldsymbol{t})\right\} \boldsymbol{d} \boldsymbol{t}
\end{aligned}
$$

Kalikan kedua ruas dengan $e^{\frac{1}{2} \frac{\cos \alpha}{\sin \alpha} \mu \omega_{1}{ }^{2}} e^{\frac{1}{2} \frac{\cos \beta}{\sin \beta} \mu \omega_{2}{ }^{2}}$, maka persamaan di atas menjadi

$$
\begin{aligned}
& e^{\frac{1}{2} \frac{\cos \alpha}{\sin \alpha} \mu \omega_{1}{ }^{2}} e^{\frac{1}{2} \frac{\cos \beta}{\sin \beta} \mu \omega_{2}{ }^{2}} F_{\mu}^{\alpha, \beta}\{f \star g\}(\boldsymbol{\omega}) \\
& =F_{\mu}^{\alpha, \beta}\left\{f_{0}(\boldsymbol{t})\right\}(\boldsymbol{\omega}) F_{\mu}^{\alpha, \beta}\{g(\boldsymbol{\varphi})\}(\boldsymbol{\omega})+\boldsymbol{i} F_{\mu}^{\alpha, \beta}\left\{f_{1}(\boldsymbol{t})\right\}(\boldsymbol{\omega}) F_{\mu}^{\alpha, \beta}\{g(\boldsymbol{\varphi})\}(\boldsymbol{\omega}) \\
& +\boldsymbol{j} F_{\mu}^{\alpha, \beta}\left\{f_{2}(\boldsymbol{t})\right\}(\boldsymbol{\omega}) F_{\mu}^{\alpha, \beta}\{g(\boldsymbol{\varphi})\}(\boldsymbol{\omega})+\boldsymbol{k} F_{\mu}^{\alpha, \beta}\left\{f_{3}(\boldsymbol{t})\right\}(\boldsymbol{\omega}) F_{\mu}^{\alpha, \beta}\{g(\boldsymbol{\varphi})\}(\boldsymbol{\omega})
\end{aligned}
$$

Kalikan kembali kedua ruas pada persamaan di atas dengan $e^{-\frac{1}{2} \frac{\cos \alpha}{\sin \alpha} \mu \omega_{1}^{2}} e^{-\frac{1}{2} \frac{\cos \beta}{\sin \beta} \mu \omega_{2}{ }^{2}}$ dan selanjutnya substitusi nilai $\boldsymbol{\varphi}=(\boldsymbol{x}-\boldsymbol{t})$, sehingga diperoleh

$$
\begin{aligned}
& F_{\mu}^{\alpha, \beta}\{f \star g\}(\boldsymbol{\omega}) \\
& =e^{-\frac{1}{2} \frac{\cos \alpha}{\sin \alpha} \mu \omega_{1}{ }^{2}} e^{-\frac{1}{2} \frac{\cos \beta}{\sin \beta} \mu \omega_{2}{ }^{2}}\left[F_{\mu}^{\alpha, \beta}\left\{f_{0}(\boldsymbol{t})\right\}(\boldsymbol{\omega}) F_{\mu}^{\alpha, \beta}\{g(\boldsymbol{x}-\boldsymbol{t})\}(\boldsymbol{\omega})\right. \\
& \\
& \quad+\boldsymbol{i} F_{\mu}^{\alpha, \beta}\left\{f_{1}(\boldsymbol{t})\right\}(\boldsymbol{\omega}) F_{\mu}^{\alpha, \beta}\{g(\boldsymbol{x}-\boldsymbol{t})\}(\boldsymbol{\omega})+\boldsymbol{j} F_{\mu}^{\alpha, \beta}\left\{f_{2}(\boldsymbol{t})\right\}(\boldsymbol{\omega}) F_{\mu}^{\alpha, \beta}\{g(\boldsymbol{x}-\boldsymbol{t})\}(\boldsymbol{\omega}) \\
& \left.\quad+\boldsymbol{k} F_{\mu}^{\alpha, \beta}\left\{f_{3}(\boldsymbol{t})\right\}(\boldsymbol{\omega}) F_{\mu}^{\alpha, \beta}\{g(\boldsymbol{x}-\boldsymbol{t})\}(\boldsymbol{\omega})\right]
\end{aligned}
$$

Dengan demikian teorema konvolusi TFFQ sisi kiri terbukti.

\section{b. Teorema 6 (TFFQ sisi kiri dari Konjugat Konvolusi)}

Misalkan $f, g \in L^{1}\left(\mathbb{R}^{2} ; \mathbb{H}\right)$ adalah dua fungsi bernilai quaternion, maka

$$
F_{\mu}^{\alpha, \beta}\{\overline{f \star g}\}(\boldsymbol{\omega})=e^{-\frac{1 \cos \alpha}{2 \sin \alpha} \mu \omega_{1}{ }^{2}} e^{-\frac{1 \cos \beta}{2 \sin \beta} \mu \omega_{2}^{2}}\left(F_{\mu}^{\alpha, \beta}\{\bar{g}(\boldsymbol{t})\}(\boldsymbol{\omega})\right)\left(F_{\mu}^{\alpha, \beta}\{\bar{f}(\boldsymbol{x}-\boldsymbol{t})\}(\boldsymbol{\omega})\right)
$$

Bukti:

Dari definisi TFFQ sisi kiri pada persamaan (1) dan teorema konvolusi TFFQ sisi kiri pada persamaan (8), diketahui

$$
\begin{aligned}
& F_{\mu}^{\alpha, \beta}\{\overline{f \star g}\}(\boldsymbol{\omega}) \\
& =\int_{\mathbb{R}^{2}} \int_{\mathbb{R}^{2}} \sqrt{\frac{1-\mu \cot \alpha}{2 \pi}} e^{\frac{1}{2} \mu\left(\frac{\cos \alpha}{\sin \alpha} x_{1}{ }^{2}-\frac{2}{\sin \alpha} x_{1} \omega_{1}+\frac{\cos \alpha}{\sin \alpha} \omega_{1}{ }^{2}\right)} \sqrt{\frac{1-\mu \cot \beta}{2 \pi}} \\
& \cdot e^{\frac{1}{2} \mu\left(\frac{\cos \beta}{\sin \beta} x_{2}{ }^{2}-\frac{2}{\sin \beta} x_{2} \omega_{2}+\frac{\cos \beta}{\sin \beta} \omega_{2}{ }^{2}\right)} \sqrt{\frac{1-\mu \cot \alpha}{2 \pi}} e^{\frac{\cos \alpha}{\sin \alpha} \mu t_{1}\left(t_{1}-x_{1}\right)} \sqrt{\frac{1-\mu \cot \beta}{2 \pi}} e^{\frac{\cos \beta}{\sin \beta} \mu t_{2}\left(t_{2}-x_{2}\right)} \\
& \cdot \bar{g}(\boldsymbol{t}) \cdot \bar{f}(\boldsymbol{x}-\boldsymbol{t}) \boldsymbol{d} \boldsymbol{t} \boldsymbol{d} \boldsymbol{x}
\end{aligned}
$$

Misalkan $\boldsymbol{\varphi}=(\boldsymbol{x}-\boldsymbol{t})$. Setelah melakukan penjabaran dan manipulasi aljabar, diperoleh $F_{\mu}^{\alpha, \beta}\{\overline{f \star g}\}(\boldsymbol{\omega})$ 


$$
\begin{aligned}
= & \sqrt{\frac{1-\mu \cot \alpha}{2 \pi}} \sqrt{\frac{1-\mu \cot \beta}{2 \pi}} \int_{\mathbb{R}^{2}} e^{\frac{1 \cos \alpha}{2 \sin \alpha} \mu t_{1}{ }^{2}} e^{\frac{1}{2} \mu\left(-\frac{2}{\sin \alpha} t_{1} \omega_{1}\right)} e^{\frac{1 \cos \beta}{2 \sin \beta} \mu t_{2}{ }^{2}} e^{\frac{1}{2} \mu\left(-\frac{2}{\sin \beta} t_{2} \omega_{1}\right)} \\
& \cdot \bar{g}(\boldsymbol{t}) \boldsymbol{d} \boldsymbol{t}\left[F_{\mu}^{\alpha, \beta}\{\bar{f}(\boldsymbol{\varphi})\}(\boldsymbol{\omega})\right]
\end{aligned}
$$

Karena tidak berlaku sifat komutatif terhadap perkalian pada bilangan quaternion, maka fungsi $\bar{g}(\boldsymbol{t})$ harus didekomposisi menjadi $\bar{g}(\boldsymbol{t})=g_{0}(\boldsymbol{t})-\boldsymbol{i} g_{1}(\boldsymbol{t})-\boldsymbol{j} g_{2}(\boldsymbol{t})-\boldsymbol{k} g_{3}(\boldsymbol{t})$, sebagai berikut $F_{\mu}^{\alpha, \beta}\{\overline{f \star g}\}(\boldsymbol{\omega})$

$$
\begin{aligned}
= & \sqrt{\frac{1-\mu \cot \alpha}{2 \pi}} \sqrt{\frac{1-\mu \cot \beta}{2 \pi}} \int_{\mathbb{R}^{2}} e^{\frac{1 \cos \alpha}{2 \sin \alpha} \mu t_{1}{ }^{2}} e^{\frac{1}{2} \mu\left(-\frac{2}{\sin \alpha} t_{1} \omega_{1}\right)} e^{\frac{1 \cos \beta}{2 \sin \beta} \mu t_{2}{ }^{2}} e^{\frac{1}{2} \mu\left(-\frac{2}{\sin \beta} t_{2} \omega_{2}\right)} \\
& \cdot\left\{g_{0}(\boldsymbol{t})-\boldsymbol{i} g_{1}(\boldsymbol{t})-\boldsymbol{j} g_{2}(\boldsymbol{t})-\boldsymbol{k} g_{3}(\boldsymbol{t})\right\}\left[F_{\mu}^{\alpha, \beta}\{\bar{f}(\boldsymbol{\varphi})\}(\boldsymbol{\omega})\right] \boldsymbol{d} \boldsymbol{t}
\end{aligned}
$$

Kalikan kedua ruas pada persamaan (4.28) dengan $e^{\frac{1 \cos \alpha}{2 \sin \alpha} \mu \omega_{1}^{2}} e^{\frac{1 \cos \beta}{2 \sin \beta} \mu \omega_{2}{ }^{2}}$. Sehingga persamaan di atas menjadi

$$
\begin{aligned}
e^{\frac{1 \cos \alpha}{2 \sin \alpha} \mu \omega_{1}{ }^{2}} e^{\frac{1 \cos \beta}{2 \sin \beta} \mu \omega_{2}{ }^{2}} F_{\mu}^{\alpha, \beta}\{\overline{f \star g}\}(\boldsymbol{\omega}) \\
=F_{\mu}^{\alpha, \beta}\left\{g_{0}(\boldsymbol{t})\right\}(\boldsymbol{\omega}) F_{\mu}^{\alpha, \beta}\{\bar{f}(\boldsymbol{\varphi})\}(\boldsymbol{\omega})-\boldsymbol{i} F_{\mu}^{\alpha, \beta}\left\{g_{1}(\boldsymbol{t})\right\}(\boldsymbol{\omega}) F_{\mu}^{\alpha, \beta}\{\bar{f}(\boldsymbol{\varphi})\}(\boldsymbol{\omega}) \\
\quad-\boldsymbol{j} F_{\mu}^{\alpha, \beta}\left\{g_{2}(\boldsymbol{t})\right\}(\boldsymbol{\omega}) F_{\mu}^{\alpha, \beta}\{\bar{f}(\boldsymbol{\varphi})\}(\boldsymbol{\omega})-\boldsymbol{k} F_{\mu}^{\alpha, \beta}\left\{g_{3}(\boldsymbol{t})\right\}(\boldsymbol{\omega}) F_{\mu}^{\alpha, \beta}\{\bar{f}(\boldsymbol{\varphi})\}(\boldsymbol{\omega})
\end{aligned}
$$

Kalikan kedua ruas pada persamaan di atas dengan $e^{-\frac{1 \cos \alpha}{2 \sin \alpha} \mu \omega_{1}{ }^{2}} e^{-\frac{1 \cos \beta}{2 \sin \beta} \mu \omega_{2}{ }^{2}}$ dan substitusikan kembali $\boldsymbol{\varphi}=\boldsymbol{x}-\boldsymbol{t}$, diperoleh

$F_{\mu}^{\alpha, \beta}\{\overline{f \star g}\}(\boldsymbol{\omega})$

$=e^{-\frac{1 \cos \alpha}{2 \sin \alpha} \mu \omega_{1}{ }^{2}} e^{-\frac{1 \cos \beta}{2 \sin \beta} \mu \omega_{2}{ }^{2}}\left(F_{\mu}^{\alpha, \beta}\{\bar{g}(\boldsymbol{t})\}(\boldsymbol{\omega})\right)\left(F_{\mu}^{\alpha, \beta}\{\bar{f}(\boldsymbol{x}-\boldsymbol{t})\}(\boldsymbol{\omega})\right)$

Dengan demikian teorema TFFQ sisi kiri dari konjugat konvolusi terbukti.

\section{c. Teorema 7 (TFFQ sisi kiri dari Translasi Salah Satu Fungsi pada Konvolusi)}

Misalkan $f, g \in L^{1}\left(\mathbb{R}^{2} ; \mathbb{H}\right)$. Jika translasi dari fungsi $f$ didefinisikan sebagai $\tau_{x_{0}} f(\boldsymbol{x})=$ $f\left(\boldsymbol{x}-\boldsymbol{x}_{\mathbf{0}}\right)$, maka TFFQ sisi kiri dari translasi salah satu fungsi pada konvolusi memenuhi $F_{\mu}^{\alpha, \beta}\left\{\tau_{x_{0}} f \star g\right\}(\boldsymbol{\omega})$

$$
\begin{aligned}
& =e^{-\frac{1 \cos \alpha}{2 \sin \alpha} \mu\left(\omega_{1}{ }^{2}-2 \omega_{1} x_{01} \cos \alpha+\left(x_{01} \cos \alpha\right)^{2}\right)} e^{\frac{1}{2} \frac{\cos \alpha}{\sin \alpha} \mu x_{01}{ }^{2}} e^{\frac{1}{2} \mu\left(-\frac{2}{\sin \alpha} x_{01} \omega_{1}\right)}
\end{aligned}
$$

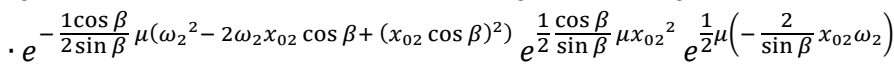

$$
\begin{aligned}
& \cdot\left[F_{\mu}^{\alpha, \beta}\left\{f_{0}\left(\boldsymbol{t}-\boldsymbol{x}_{\mathbf{0}}\right)\right\}\left(\omega_{1}-x_{01} \cos \alpha, \omega_{2}-x_{02} \cos \beta\right) F_{\mu}^{\alpha, \beta}\{g\}(\boldsymbol{x}-\boldsymbol{t})\right. \\
& +\boldsymbol{i} F_{\mu}^{\alpha, \beta}\left\{f_{1}\left(\boldsymbol{t}-\boldsymbol{x}_{\mathbf{0}}\right)\right\}\left(\omega_{1}-x_{01} \cos \alpha, \omega_{2}-x_{02} \cos \beta\right) F_{\mu}^{\alpha, \beta}\{g\}(\boldsymbol{x}-\boldsymbol{t}) \\
& +\boldsymbol{j} F_{\mu}^{\alpha, \beta}\left\{f_{2}\left(\boldsymbol{t}-\boldsymbol{x}_{\mathbf{0}}\right)\right\}\left(\omega_{1}-x_{01} \cos \alpha, \omega_{2}-x_{02} \cos \beta\right) F_{\mu}^{\alpha, \beta}\{g\}(\boldsymbol{x}-\boldsymbol{t}) \\
& \left.+\boldsymbol{k} F_{\mu}^{\alpha, \beta}\left\{f_{3}\left(\boldsymbol{t}-\boldsymbol{x}_{\mathbf{0}}\right)\right\}\left(\omega_{1}-x_{01} \cos \alpha, \omega_{2}-x_{02} \cos \beta\right) F_{\mu}^{\alpha, \beta}\{g\}(\boldsymbol{x}-\boldsymbol{t})\right]
\end{aligned}
$$

Bukti :

Dari definisi TFFQ sisi pada persamaan (1), diperoleh

$$
\begin{aligned}
& F_{\mu}^{\alpha, \beta}\left\{\tau_{x_{0}} f \star g\right\}(\boldsymbol{\omega}) \\
& =\int_{\mathbb{R}^{2}} \int_{\mathbb{R}^{2}} \sqrt{\frac{1-\mu \cot \alpha}{2 \pi}} e^{\frac{1}{2} \mu\left(\frac{\cos \alpha}{\sin \alpha} x_{1}{ }^{2}-\frac{2}{\sin \alpha} x_{1} \omega_{1}+\frac{\cos \alpha}{\sin \alpha} \omega_{1}{ }^{2}\right)} \sqrt{\frac{1-\mu \cot \beta}{2 \pi}} \\
& \cdot e^{\frac{1}{2} \mu\left(\frac{\cos \beta}{\sin \beta} x_{2}{ }^{2}-\frac{2}{\sin \beta} x_{2} \omega_{2}+\frac{\cos \beta}{\sin \beta} \omega_{2}{ }^{2}\right)} \sqrt{\frac{1-\mu \cot \alpha}{2 \pi}} e^{\frac{\cos \alpha}{\sin \alpha} \mu t_{1}\left(t_{1}-x_{1}\right)} \sqrt{\frac{1-\mu \cot \beta}{2 \pi}} e^{\frac{\cos \beta}{\sin \beta} \mu t_{2}\left(t_{2}-x_{2}\right)}
\end{aligned}
$$


- $\tau_{x_{0}} f(\boldsymbol{t}) g(\boldsymbol{x}-\boldsymbol{t}) \boldsymbol{d t} \boldsymbol{d x}$

Misalkan $\boldsymbol{\varphi}=\boldsymbol{x}-\boldsymbol{t}$. Setelah dilakukan proses manipulasi aljabar, dengan mudah diperoleh $F_{\mu}^{\alpha, \beta}\left\{\tau_{x_{0}} f \star g\right\}(\boldsymbol{\omega})$

$=\left[\sqrt{\frac{1-\mu \cot \alpha}{2 \pi}} \sqrt{\frac{1-\mu \cot \beta}{2 \pi}} \int_{\mathbb{R}^{2}} e^{\frac{1 \cos \alpha}{2 \sin \alpha} \mu t_{1}{ }^{2}} e^{\frac{1}{2} \mu\left(-\frac{2}{\sin \alpha} t_{1} \omega_{1}\right)} e^{\frac{1 \cos \beta}{2 \sin \beta} \mu t_{2}{ }^{2}} e^{\frac{1}{2} \mu\left(-\frac{2}{\sin \beta} t_{2} \omega_{2}\right)}\right.$ $\left.\cdot f\left(\boldsymbol{t}-\boldsymbol{x}_{\mathbf{0}}\right) \boldsymbol{d} \boldsymbol{t}\right] F_{\mu}^{\alpha, \beta}\{g\}(\boldsymbol{\varphi})$

Misalkan $z=t-x_{0}$, maka

$$
\begin{aligned}
& F_{\mu}^{\alpha, \beta}\left\{\tau_{x_{0}} f \star g\right\}(\boldsymbol{\omega}) \\
& =\left[\int_{\mathbb{R}^{2}} \sqrt{\frac{1-\mu \cot \alpha}{2 \pi}} \sqrt{\frac{1-\mu \cot \beta}{2 \pi}} e^{\frac{1 \cos \alpha}{2 \sin \alpha} \mu\left(z_{1}+x_{01}\right)^{2}} e^{\frac{1}{2} \mu\left(-\frac{2}{\sin \alpha}\left(z_{1}+x_{01}\right) \omega_{1}\right)} e^{\frac{1 \cos \beta}{2 \sin \beta} \mu\left(z_{2}+x_{02}\right)^{2}}\right. \\
& \left.\cdot e^{\frac{1}{2} \mu\left(-\frac{2}{\sin \beta}\left(z_{2}+x_{02}\right) \omega_{2}\right)} f(\mathbf{z}) \boldsymbol{d z}\right] F_{\mu}^{\alpha, \beta}\{g\}(\boldsymbol{\varphi})
\end{aligned}
$$

Karena tidak berlaku sifat komutatif terhadap operasi perkalian pada quaternion, maka fungsi $f(\mathbf{z})$ didekomposisi menjadi $f(\mathbf{z})=f_{0}(\mathbf{z})+\boldsymbol{i} f_{1}(\mathbf{z})+\boldsymbol{j} f_{2}(\mathbf{z})+\boldsymbol{k} f_{3}(\mathbf{z})$, diperoleh

$$
\begin{aligned}
& F_{\mu}^{\alpha, \beta}\left\{\tau_{x_{0}} f \star g\right\}(\boldsymbol{\omega}) \\
& =\left[\sqrt{\frac{1-\mu \cot \alpha}{2 \pi}} \sqrt{\frac{1-\mu \cot \beta}{2 \pi}} \int_{\mathbb{R}^{2}} e^{\frac{1}{2} \mu\left(\frac{\cos \alpha}{\sin \alpha} z_{1}{ }^{2}-\frac{2}{\sin \alpha} z_{1}\left(\omega_{1}-x_{01} \cos \alpha\right)\right)} e^{\frac{1}{2} \frac{\cos \alpha}{\sin \alpha} \mu x_{01}{ }^{2}}\right. \\
& \cdot e^{\frac{1}{2} \mu\left(-\frac{2}{\sin \alpha} x_{01} \omega_{1}\right)} e^{\frac{1}{2} \mu\left(\frac{\cos \beta}{\sin \beta} z_{2}{ }^{2}-\frac{2}{\sin \beta} z_{2}\left(\omega_{2}-x_{02} \cos \beta\right)\right)} e^{\frac{1}{2} \frac{\cos \beta}{\sin \beta} \mu x_{02}{ }^{2}} e^{\frac{1}{2} \mu\left(-\frac{2}{\sin \beta} x_{02} \omega_{2}\right)} \\
& \left.\cdot\left\{f_{0}(\mathbf{z}) F_{\mu}^{\alpha, \beta}\{g\}(\boldsymbol{\varphi})+\boldsymbol{i} f_{1}(\mathbf{z}) F_{\mu}^{\alpha, \beta}\{g\}(\boldsymbol{\varphi})+\boldsymbol{j} f_{2}(\mathbf{z}) F_{\mu}^{\alpha, \beta}\{g\}(\boldsymbol{\varphi})+\boldsymbol{k} f_{3}(\mathbf{z}) F_{\mu}^{\alpha, \beta}\{g\}(\boldsymbol{\varphi})\right\} \boldsymbol{d} \mathbf{z}\right]
\end{aligned}
$$

Kalikan kedua ruas dengan $e^{\frac{1 \cos \alpha}{2 \sin \alpha} \mu\left(\omega_{1}-x_{01} \cos \alpha\right)^{2}} e^{\frac{1 \cos \beta}{2 \sin \beta} \mu\left(\omega_{2}-x_{02} \cos \beta\right)^{2}}$ dan lakukan manipulasi aljabar pada persamaan tersebut sehingga menjadi

$$
\begin{aligned}
& e^{\frac{1 \cos \alpha}{2 \sin \alpha} \mu\left(\omega_{1}-x_{01} \cos \alpha\right)^{2}} e^{\frac{1 \cos \beta}{2 \sin \beta} \mu\left(\omega_{2}-x_{02} \cos \beta\right)^{2}} F_{\mu}^{\alpha, \beta}\left\{\tau_{x_{0}} f \star g\right\}(\boldsymbol{\omega}) \\
& =e^{\frac{1}{2} \frac{\cos \alpha}{\sin \alpha} \mu x_{01}{ }^{2}} e^{\frac{1}{2} \mu\left(-\frac{2}{\sin \alpha} x_{01} \omega_{1}\right)} e^{\frac{1}{2} \frac{\cos \beta}{\sin \beta} \mu x_{02}{ }^{2}} e^{\frac{1}{2} \mu\left(-\frac{2}{\sin \beta} x_{02} \omega_{2}\right)} \\
& \text {. }\left[F_{\mu}^{\alpha, \beta}\left\{f_{0}(\mathbf{z})\right\}\left(\omega_{1}-x_{01} \cos \alpha, \omega_{2}-x_{02} \cos \beta\right) F_{\mu}^{\alpha, \beta}\{g\}(\boldsymbol{\varphi})\right. \\
& +\boldsymbol{i} F_{\mu}^{\alpha, \beta}\left\{f_{1}(\mathbf{z})\right\}\left(\omega_{1}-x_{01} \cos \alpha, \omega_{2}-x_{02} \cos \beta\right) F_{\mu}^{\alpha, \beta}\{g\}(\boldsymbol{\varphi}) \\
& +\boldsymbol{j} F_{\mu}^{\alpha, \beta}\left\{f_{2}(\mathbf{z})\right\}\left(\omega_{1}-x_{01} \cos \alpha, \omega_{2}-x_{02} \cos \beta\right) F_{\mu}^{\alpha, \beta}\{g\}(\boldsymbol{\varphi}) \\
& \left.+\boldsymbol{k} F_{\mu}^{\alpha, \beta}\left\{f_{3}(\mathbf{z})\right\}\left(\omega_{1}-x_{01} \cos \alpha, \omega_{2}-x_{02} \cos \beta\right) F_{\mu}^{\alpha, \beta}\{g\}(\boldsymbol{\varphi})\right]
\end{aligned}
$$

Kalikan kedua ruas dengan $e^{-\frac{1 \cos \alpha}{2 \sin \alpha} \mu\left(\omega_{1}-x_{01} \cos \alpha\right)^{2}} e^{-\frac{1 \cos \beta}{2 \sin \beta} \mu\left(\omega_{2}-x_{02} \cos \beta\right)^{2}}$ serta substitusi kembali nilai $\boldsymbol{\varphi}=(\boldsymbol{x}-\boldsymbol{t})$ dan $\boldsymbol{z}=\boldsymbol{t}-\boldsymbol{x}_{\mathbf{0}}$, sehingga persamaan di atas menjadi

$$
\begin{aligned}
& F_{\mu}^{\alpha, \beta}\left\{\tau_{x_{0}} f \star g\right\}(\boldsymbol{\omega}) \\
& =e^{-\frac{1 \cos \alpha}{2 \sin \alpha} \mu\left(\omega_{1}{ }^{2}-2 \omega_{1} x_{01} \cos \alpha+\left(x_{01} \cos \alpha\right)^{2}\right)} e^{\frac{1}{2} \frac{\cos \alpha}{\sin \alpha} \mu x_{01}{ }^{2}} e^{\frac{1}{2} \mu\left(-\frac{2}{\sin \alpha} x_{01} \omega_{1}\right)} \\
& \cdot e^{-\frac{1 \cos \beta}{2 \sin \beta} \mu\left(\omega_{2}{ }^{2}-2 \omega_{2} x_{02} \cos \beta+\left(x_{02} \cos \beta\right)^{2}\right)} e^{\frac{1}{2} \frac{\cos \beta}{\sin \beta} \mu x_{02}{ }^{2}} e^{\frac{1}{2} \mu\left(-\frac{2}{\sin \beta} x_{02} \omega_{2}\right)} \\
& \quad \cdot\left[F_{\mu}^{\alpha, \beta}\left\{f_{0}\left(\boldsymbol{t}-\boldsymbol{x}_{\mathbf{0}}\right)\right\}\left(\omega_{1}-x_{01} \cos \alpha, \omega_{2}-x_{02} \cos \beta\right) F_{\mu}^{\alpha, \beta}\{g\}(\boldsymbol{x}-\boldsymbol{t})\right. \\
& \quad+\boldsymbol{i} F_{\mu}^{\alpha, \beta}\left\{f_{1}\left(\boldsymbol{t}-\boldsymbol{x}_{\mathbf{0}}\right)\right\}\left(\omega_{1}-x_{01} \cos \alpha, \omega_{2}-x_{02} \cos \beta\right) F_{\mu}^{\alpha, \beta}\{g\}(\boldsymbol{x}-\boldsymbol{t}) \\
& \quad+\boldsymbol{j} F_{\mu}^{\alpha, \beta}\left\{f_{2}\left(\boldsymbol{t}-\boldsymbol{x}_{\mathbf{0}}\right)\right\}\left(\omega_{1}-x_{01} \cos \alpha, \omega_{1}-x_{02} \cos \beta\right) F_{\mu}^{\alpha, \beta}\{g\}(\boldsymbol{x}-\boldsymbol{t})
\end{aligned}
$$




$$
\left.+\boldsymbol{k} F_{\mu}^{\alpha, \beta}\left\{f_{3}\left(\boldsymbol{t}-\boldsymbol{x}_{\mathbf{0}}\right)\right\}\left(\omega_{1}-x_{01} \cos \alpha, \omega_{2}-x_{02} \cos \beta\right) F_{\mu}^{\alpha, \beta}\{g\}(\boldsymbol{x}-\boldsymbol{t})\right]
$$

Dengan demikian teorema TFFQ sisi kiri dari tranlasi konvolusi dua fungsi quaternion terbukti.

\section{KESIMPULAN}

Berdasarkan hasil dan pembahasan yang telah dilakukan, pada penelitian ini berhasil dirumuskan definisi TFFQ sisi kiri dan sifat-sifatnya (linearitas, translasi, modulasi dan skala). Selain itu, dirumuskan pula teorema konvolusi pada TFFQ sisi kiri serta TFFQ sisi kiri dari konjugat konvolusi dan translasi salah satu fungsi pada konvolusi.

\section{DAFTAR PUSTAKA}

[1] Almeida, L B, IEEE Transactions on Signal Processing, The Fractional Fourier Transform and Time-Frequency Representations, 42, 1994, 3084-3091.

[2] Guanlei, X, Xiaotong, W, and Xiaogang, X, Signal Processing, Fractional Quaternion Fourier Transform, Convolution and Correlation, 88, 2008, 2511-2517.

[3] Hitzer, E M S, Advances in Applied Clifford Algebras, Quaternion Fourier Transform on Quaternion Fourier Generalizations, 17, 2007, 497-517.

[4] Irwan, M, Jurnal Matematika dan Statistika serta Aplikasinya, Quaternion and It's Properties, 3, 2015, 16-20.

[5] Morais, J P, Georgiev S, and Sprobig, W, Real Quaternionic Calculus Handbook, Springer Basel, New York, 1-8.

[6] Pei, S C, Ding, J J, and Chang, J H, IEEE Transactions on Signal Processing, Efficient Implementation of Quaternion Fourier Transform, Convolution and Correlation by 2-D Complex FFT, 49, 2001, 2783-2797.

[7] Sangkala, N S, Konvolusi Transformasi Fourier Fraksional Quaternion Sisi Kanan dan Sifatsifatnya. Universitas Hasanuddin, Makassar.

[8] Saribulut, L, Teke, A, and Tumay, M, Jurnal of Electrical and Electronics Engineering Research, Fundamentals and Literature Review of Fourier Transform in Power Quality Issues, 5, 2013, 922.

[9] Sharma, V D, and Deshmukh, P B, International Journal of Engineering Science and Research Technology, Convolution Structure Fractional Quaternion Fourier Transform, 5, 2016, 1463 1467. 
[10] Wei, D, and Li Y, International Journal for Light and Electron Optics, Different Forms of Plancherel Theorem for Fractional Quaternion Fourier Transform, 124, 2013, 6999-7002.

[11] Yetik, I S, Kutay, M A, and Ozaktas, H M, Proceedings of Design Engineering Technical Conferences, The Fractional Fourier Transform and Its Applications to Image Representation and Beamforming, 2003, 771-780. 